\title{
Index for Volume 12 of Molecular Plant-Microbe Interactions
}

\section{AUTHOR AND SUBJECT INDEX}

Abad, P., 585

Abderhaiden, O., 647

Adam, L., 1051

Agrobacterium rhizogenes, to promote nodulation, 275

Albersheim, P., 703

Albizia julibrissin, nodulation by Rhizobium, cover photo, April

Alfalfa

-anthracnose, nonpathogenic mutants and kinase, 430

-nodulation, aspartate aminotransferase role, 263

-nodulation, nitrogen and carbon metabolism, gene map, 526

-nodulation affected by Serratia marcescens chitinase gene, 748

—nodulation by Sinorhizobium meliloti, gene fusion, cover photo, July

- protein kinase homolog, mitogen-activated, TDY1 gene, 882

-Rhizobium spp. on, fusicoccin role in infection, 1090

Alfalfa mosaic virus, movement protein, endoplasmic reticulum, onion epidermal cells, 680

Alfano, F., 275

Allen, K. D., 328

Alternaria alternata, on Japanese pear

-AK-toxin biosynthesis, insertional mutagenesis, 691

- melanin biosynthesis gene, disruption, 59

Andersson, R. A., 575

Arabidopsis thaliana

-cauliflower mosaic virus, gene expression, CaMV gene VI transgene, 377

—disease resistant mutant, 1031

- downy mildew, mapping of RPP13 locus, 792

— gene change, rhizobacterium induction, 951

- powdery mildew, species comparison and resistance, 1051

-Pseudomonas syringae, resistance and locus identification, 911

-rhizosphere-colonizing biocontrol bacteria, 720

-salicylic acid induction, regulation, 1022

-turnip mosaic virus, resistance, 1016

Aristizábal, F. A., 440

Arredondo-Peter, R., 1008

Arthaud, L., 585

Aspergillus niger, polygalacturonases from, 703

Atzorn, R., 74

Audenaert, K., 450

Auxin, reduction in Vicia sativa roots, lipochitin oligosaccharide effect, 839

Avrova, A. O., 356, 1114

Azoarcus sp.

-on rice, root colonization, cover photo, September

—on rice roots, endophytic expression of nif genes, 813

Azorhizobium caulinodans

-lipochitooligosaccharide biosynthesis in, 68

-on Phaseolus vulgaris, Nod factors and

chemical substituents, 820

Baillieul, F., 655

Bairl, A., 218

Bakker, J., 197, 585, 872

Balázs, E., 1105

Balestrini, R., 785, 862

Balhadère, P. V., 129

Ballance, G. M., 728

Ballio, A., 391, 401

Barker, D. G., 604

Barley

-brome mosaic virus on, temperature-

dependent mechanism, 615

- powdery mildew, susceptibility to rice blast, 508

Barthels, N., 440

Baulcombe, D. C., 197, 536

Baum, T. J., 64, 585, 663

Bauw, G., 862

Beachy, R. N., 143, 476

Bean dwarf mosaic virus, cell-to-cell and longdistance movement, hosts and nonhosts, 345

Bellés, J. M., 227

Bellogin, R. A., 207

Bendahmane, A., 197

Bendahmane, M., 143, 476

Bender, C. L., 563

Bentley, S. D., 499

Bergmann, C. W., 703

Bernhart, I., 391, 401

Bernier, L., 6

Bertioli, D. J., 189

Bertolla, F., 467

Beynon, J., 792

Bindslev, L., 960

Birch, P. R. J., 356, 1114

Bisseling, T., 829

Bittner-Eddy, P., 792

Bjourson, A. J., 825

Blaylock, L. A., 171

Blumeria graminis, on barley, mutation conferring resistance, 508

Bol, J. F., 720

Bonfante, P., 785, 862

Bonnema, G., 93

Boot, K. J. M., 839

Borja, M., 87, 153

Botrytis cinerea

-in chestnut, cystatin antagonism, 624

-on potato, snakin-1 effect on, 16

Boulton, M. I., 894

Bourque, J. E., 919

Bradyrhizobium spp.

-B. elkanii, rhizobitoxine role, legume symbiosis, 1082

-B. japonicum: on soybean, 53-kDa nodulin control, 218; on Vigna umbellata, nodulation, mutant, signals, 766

Brasier, C. M., 6

Brassica napus, blackleg, defense gene expression, 410

Bravo, M., 35

Brewin, N. J., 785

Brito, B., 467

Brome mosaic virus, on barley, temperaturedependent mechanism, 615

Broughton, W. J., 293

Bucciarelli, B., 526

Buchala, A., 450

Bülow, L., 182

Burrows, P. R., 189

Callahan, T. M., 901

Can, C., 792

Candresse, T., 337, 367

Canto, T., 733, 985

Capieau, K., 450

Caporgno, J., 35

Capsicum аппиит

- anthracnose, cytochrome P450 gene, 1042

-Xanthomonas campestris, pathogenesis-

related cDNA genes, 0751

Caputo, E., 24, 926

Carlson, R., 766

Castañeda, A., 556

Castillo, A., 45

Cauliflower mosaic virus

- 35S promoter and subdomains, nematoderesponsive activity, 189

-in Arabidopsis, gene expression and CaMV gene VI transgene, 377

-on Nicotiana spp., symptoms, cover photo, October

- on Nicotiana clevelandii, gene interaction and cell death, 919

Cecchini, E., 377

Cerbah, M., 947

Cercospora kikuchii, on soybean, cercosporin transporter, requirement, 901

Cerff, R., 182

Cermola, M., 515, 926

Chamaecrista fasciculata, nodulation by Rhizobium, 293

Chen, X.-Y., 1095

Chestnut, cystatin, antifungal activity, 624

Chet, I., 1000

Chiurazzi, M., 275

Cho, M. J., 143, 476

Cho, Y., 633

Chu, M., 285

Ciesiolka, L. D., 35

Citrullus lanatus, Colletotrichum magna on, pathogenicity conversion by gene disruption, 969

Citrus exocortis viroid, on tomato, gentisic acid effect, 227

Cladosporium fulvum, on tomato

- Cf-9 resistance gene, homologues, 93

-Tnt1 promoter retrotransposon, 592 
Clavibacter michiganensis, on potato, snakin-1 effect on, 16

Clay, R. P., 703Clive Lo, S.-C., 479

Cochliobolus spp.

-C. heterostrophus, on sorghum, Colletotrichum sublineolum comparison, 479

-C. sativus, polygalacturonases from, 703

Cohen, S., 774

Cohn, J., 766

Cole, A. B., 919

Colletotrichum spp.

-C. gloeosporioides, on pepper, cytochrome P450 gene, 1042

-C. graminicola, in chestnut, cystatin antagonism, 624

-C. lindemuthianum: on Phaseolus vulgaris, coevolution, ancestral resistance gene cluster, 774; polygalacturonases from, 703

-C. magna on watermelon, pathogenicity conversion, gene disruption, 969

-C. sublineolum, on sorghum, Cochliobolus heterostrophus comparison, defense-related genes, 479

-C. trifolii, on alfalfa, nonpathogenic mutants, kinase, 430

Condemine, G., 45

Conejero, V., 227

Conover, S. M., 35

Cook, B. J., 703

Cooper, J. E., 825

Cordelier, S., 655

Corvera, A., 236

Costet, L., 655

Cotton, cells treated with fungal elicitors, gene pattern, 1095

Cover photo

- alfalfa nodulation by Sinorhizobium meliloti, gene fusion, July

—Bradyrhizobium elkanii on soybean, foliar chlorosis, December

-cauliflower mosic virus, on tobacco, symptoms, October

-Erwinia amylovora on apple, iron transport system, May

-Erysiphe graminis on wheat, cell invasion, August

- Heterodera glycines on soybean roots, immunofluorescence, January

-Paenibacillus polymyxa on Arabidopsis thaliana, November

- panicum mosaic virus on pearl millet panicles, February

- Rhizobium etli on Phaseolus vulgaris, nodules and promoters, June

-Rhizobium inoculation of Albizia julibrissin, Tephrosia vogelii, Psophocarpus tetragonolobus and Sophora davidii, nodulation pattern, April

—rice root colonized with Azoarcus sp., September

— tobacco cyst nematode, March

Covey, S. N., 377

Crockard, M. A., 825

Crute, I., 792

Cryphonectria parasitica, polygalacturonases from, 703

Cubo, T., 207

Cucumber green mottle mosaic virus, in cucumber phloem, 112

Cucumber mosaic virus

- on Nicotiana glutinosa, stunting and amino acid in coat protein, 1105

—replicase-mediated resistance, 743 —on squash, cell-to-cell movement and capsid protein sequence, 628

-tubule generation, 3a protein, 985

Culley, D., 410

Culver, J. N., 247

Dahiya, P., 785

Dangl, J. L., 1022

Dardick, C. D., 247

Darvill, A. G., 703

Davies, J. W., 894

Davis, E. L., 64, 663

Day, R. B., 766

De Bellis, R., 862

de Billy, F., 544

De Boer, J. M., 585, 663

de Carvalho, D., 862

de Groot, K., 459

De Jong, W., 536, 1114

del Campo, F. F., 440

Dellagi, A., 463

Della Serra, M., 391, 401

Della Volpe, C., 391

Delonix regia, nodulation by Rhizobium, 293

Del Sorbo, G., 419

deLuca, N. G., 994

De Meutter, J., 440

De Meyer, G., 450

de Oliveira, J. C. F., 774

de Ruijter, N. C. A., 829

Desvoyes, B., 670

De Wit, P. J. G. M., 459, 592

D'Haeze, W., 68

Dickman, M. B., 430

Dietrich, R. A., 1022

Di Giorgio, D., 391, 401

Ding, X. S., 143, 476, 615

Donaire, J. P., 1090

Donzelli, B., 419

Dooner, H. K., 1031

Dorey, S., 655

Draper, J., 74

Dron, M., 774

Duan, Y. P., 556

Dudler, R., 647

Duncan, J. M., 356

Dunez, J., 337

Dunlap, J., 766

Duodu, S., 1082

Düring, K., 182

Dusha, I., 755

Egener, T., 813

Ehrenshaft, M., 901

Elad, Y., 1000

Ellwood, S., 1051

Elm, Dutch elm disease, pathogenicity gene and introgression, 6

Emons, A. M. C., 829

Enard, C., 45

Erdei, L., 755

Erdos, G., 556

Errata

— vol. 11, no. 11, 1998, 84

-vol. 11 , no. $12,1998,168$

- vol. 12, no. 2, 1999, 476

Erwinia spp.

-E. amylovora: on apple, iron transport system, cover photo, May; ferrioxamine receptor gene, pathogenesis expression, 463

-E. carotovora: on Arabidopsis thaliana, jasmonic acid syntheis, cell-wall-degrading enzymes, 640; on potato, gene mutation and virulence, 499; on potato tuber, maize
GapC4 promoter, 182; on tobacco, virulence and response regulator ExpM, 575

- E. chrysanthemi: exopolysaccharide synthesis and virulence factor, repressor coregulation, 45; fur repressor protein role, iron effect, 119; pectate lyase induction, pir gene regulation, 385 ; on potato tuber, pectinase gene expression, 845

Erysiphe spp., on Arabidopsis thaliana, species comparison and resistance, 1051

- E. graminis, on barley, conidial differentiation, cAMP and protein kinase A involvement, 960; on wheat, defense-related genes and transient assay system, 647; on wheat, epidermal cell invasion, cover photo, August; on wheat, systemic acquired resistance, 53

Escobar, C., 440

Espuny, R., 207

Esser, E., 252

Et-Touil, A., 6

Expert, D., 119, 463

Fagiuoli, G., 391

Favery, B., 585

Favre, R., 515, 926

Fayos, J., 227

Fedorova, M., 882

Fenoll, C., 440

Fernandez, I., 337

Figura, I., 845

Flasinski, S., 615

Fong, S. H. T., 1064

Forsyth, A., 536

Foster, A. J., 129

Franken, P., 934

Franza, T., 119

Fristensky, B., 410

Fritig, B., 655

Frostegård, Å., 467

Füller, P., 934

Fusarium spp.

- F. moniliforme, polygalacturonases from, 703

$-F$. oxysporum, tomatinase from, new saponinase class, 852

Gabriel, D. W., 556

Galili, S., 1000

Gamas, P., 544

Gantt, J. S., 263, 882

García, J. A.., 103

García-Arenal, F., 112

García-Olmedo, F., 16

Garro, R., 227

Gasverde, E., 785

Gearids, J. D., 35

Geffroy, V., 774

Gelvin, S., 835

Gene

- avrRxv, in tomato, expression regulation, 35

- BRM2, in Japanese pear, melanin

biosynthesis, 59

-cDNA, on pepper, pathogenesis relation, 0751

— dnaK, Pseudomonas syringae on soybean, heat stress, 563

- metZ, in Rhizobium etli, role in bean, 24

—nodX, in Rhizobium leguminosarum strains, 252

-pathogenicity, in Ophiostoma novo-ulmi, 6

- pectinase, in potato tubers, 845

- pepCYP, pepper and anthracnose interaction, 1042 
-StSN1, in potato, pathogen activity, 16

- TDY1, in alfalfa, protein kinase homolog, 882

— trpB, in Rhizobium etli, on bean, 926

Gentisic acid, on tomato, salicylic acid comparison, 227

Gepts, P., 774

Geri, C., 377

Gheysen, G., 440

Gianinazzi, S., 976

Gianinazzi-Pearson, V., 976

Giannakou, M. E., 377

Gil, A., 207

Gilbertson, R. L., 337

Ginzberg, I., 1000

Globodera spp.

- G. pallida, on potato, resistance in wild species, 197

-G. rostochiensis, tobacco leaf protoplast proliferation by, human blood cells, 872

-G. tabacum: on tobacco, cover photo, March; on transgenic tobacco, glucuronidase assay, 189

Glomus spp.

- G. intraradices, on tobacco, disease severity change induced by, 1000

-G. mosseae: on pea, defense genes induced by, 976; rhizobacterial, molecular characterization, 934

-G. versiforme: on Medicago truncatula symbiosis, novel genes, 171; on pea, lectinlike glycoprotein, gene encoding, 785

Glycine max (see Soybean)

Görlach, J., 53

Govers, F., 459

Goverse, A., 872

Grandbastien, M.-A., 592

Grapevine chrome mosaic virus, on Nicotiana spp., necrotic response, 337

Gregerson, R. G., 263, 882

Gresshoff, P. M., 275

Grosjean, C., 544

Guldmann, L.-L., 785

Gunn, N., 792

Guo, H. S., 103

Gurr, S. J., 960

Hadwiger, L. A., 410

Hall, A. A., 960

Handley, V., 35

Harman, G. E., 419

Harrison, J. J., 171

He, C., 1064

Hehl, R., 182

Heilbronn, J., 1114

Heinstein, P., 797

Heinz, E. B., 803

Helder, J., 872

Hernández, G., 1008

Herrera, D. M., 1016

Heterodera glycines

-endoglucanase secretion, localization, 64

-on Glycine max, gene isolation and transcription, polygalacturonase, 490

-on soybean, endoglucanases in esophageal glands, 663

- on soybean roots, immunofluorescence, cover photo, January

Heu, S., 633

Hipskind, J. D., 479

Hoevens, K. C. M., 263

Höfte, M., 450

Holsters, M., 68

Holub, E. B., 792
Honée, G., 459

Huang, M., 680

Hughes, D., 766

Hugouvieux-Cotte-Pattat, N., 845

Huguet, T., 544

Hurek, T., 813

Hussey, R. S., 64, 585, 663

Hwang, B. K., 0751

Hwin, T., 35

Hyman, L. J., 499

Hypersensitivity

-in bean, Pseudomonas syringae and tobacco necrosis virus, calcium-binding protein, 712

-Cladosporium fulvum and Phytophthora

infestans on tomato and tobacco, 459

- Phytophthora infestans on potato, 356

— tobacco to Pseudomonas syringae, jasmonic acid, 74

-tobacco mosaic virus, localized acquired resistance and salicylic acid, 655

- tobacco mosaic virus in pepper, eggplant, and tobacco, 247

-Xanthomonas campestris, in nonhosts, 633

Iaccarino, M., 24, 275, 515

International Congress of Molecular PlantMicrobe Interactions, meeting summary in Amsterdam, 835

Jackson, A. O., 153, 87

Jafra, S., 845

Jakobek, J. L., 712

Jarosch, B., 508

Johnston, A. W. B., 994

Jones, D. A., 93

Jones, J. D. G., 93

Journet, E.-P., 604

Jung, H. W., 0751

Kamoun, S., 459

Kanyuka, K., 197

Kapulnik, Y., 1000

Kauffman, S., 655

Kavanagh, T. A., 536

Kavelaars, A., 872

Kawamura, C., 59

Kee, H.-K., 1042

Keen, N., 835

Keith, L. M. W., 563

Kenton, P., 74

Kevei, Z., 947

Khan, R., 733

Kijne, J. W., 839

Kim, K. S., 1042

Kim, K. Y., 748

Kim, Y. S., 1042

Király, L., 919

Klein-Lankhorst, R., 197

Klessig, D. F., 1031

Kliebenstein, D. J., 1022

Knap, H. T., 490

Knoester, M., 720

Ko, M. K., 1042

Kogel, K.-H., 508

Köhler, U., 182

Kolli, V. K., 766

Kondorosi, Á., 755, 947

Kondorosi, E., 947

Kooman-Gersmann, M., 459

Kostenyuk, I., 1042

Krishnan, A. H., 748

Krishnan, H. B., 207, 748

Kubicek, C. P., 419

Kuecker, J., 670
Laeremans, T., 820

Lamari, L., 728

Lambert, K. N., 328

Lamberti, A., 275

Lamrabet, Y., 207

Langin, T., 774

Last, R. L., 1022

Laugé, R., 459

Laurent, P., 862

Le Gall, O., 337, 367

Leptosphaeria maculans, in canola, defense gene expression, 410

Lim, C. O., 143, 476

Linder, D., 218

Linder, M., 218

Lindgren, P. B., 712

Liu, C.-J., 1095

Liu, H., 894

Logkowska, E., 845

Long, S., 835

López-Moya, J. J., 103

López-Solanilla, E., 624

Lorito, M., 419

Lotus japonicus, nodulation- and root-related genes, T-DNA tagging, 275

Lu, B., 247

Lucas, W. J., 337

Lugtenberg, B. J. J., 252

Lyon, G. D., 356, 1114

Mach, R., 419

Madueño, F., 16

Magnaporthe grisea

-on barley, mutation conferring susceptibility, 508

-on rice, pathogenicity mutants, insertional mutagenesis, 129

-on rice, wounding and infection, kinase induction, 1064

Mahalingam, R., 490

Maize streak virus, coat protein, viral DNA nuclear transport, 894

Malcuit, I., 536

Maldonado-Mendoza, I. E., 171

Marano, M. R., 536

Mariën, J., 820

Martin, A. C., 1022

Martín, A. M., 1016

Martin, F., 862

Martínez-Romero, E., 236, 820

Martirani, L., 275

Mathis, R., 544

Maule, A. J., 367

May, A. G., 994

Meade, M. J., 901

Medicago truncatula

-FISH chromosome mapping, karyotype analysis, 947

-Glomus versiforme symbiosis, novel genes, 171

-root cortical cell activation, Nod factorinducible molecular marker, 604

-Sinorhizobium meliloti on roots, early nodulin gene, 544

Megias, M., 207

Meloidogyne spp

-M. incognita: expression analysis of plant parasitism, endoglucanase and cDNA, 585; on tomato, LEMMI9 gene and promoter analysis, 440; on transgenic tobacco, glucuronidase assay, 189

- M. javanica, chorismate mutase, gland specific, 328 
Menestrina, G., 391, 401

Merrick, M., 515

Métraux, J.-P., 450

Meyers, D., 64

Mhiri, C., 592

Milner, J. J., 377

Minsavage, G. V., 35

Mirabella, R., 275

Molina, A., 16, 53

Mooijman, P., 197

Mora, J., 1008

Moreno, M., 16

Mulholland, V., 499

Müller, P., 218

Mur, L. A. J., 74

Mycorrhizae, Glomus versiforme on Medicago truncatula, novel genes, 171

Nasser, W., 385

Navarro, P., 227

Nectria haematococca, on pea, regulatory signals in culture and pathogenesis, 733

Neema, C., 774

Nehls, U., 862

Nelson, R. S., 143, 476, 615

Nesme, X., 467

Nicandra physaloides, potato virus A, systemic infection determinants, 1074

Nicholson, R. L., 479

Nicotiana spp. (see also Tobacco): cauliflower mosaic virus, gene interaction and systemic cell death, 919; grapevine chrome mosaic virus, necrotic response, 337

$-N$. benthamiana: infection sites for tobacco mosaic virus, 143, 476; plum pox virus, resistance and mitotic stability, transgene silencing, 103; tomato bushy stunt virus on, wild-type virus restoration, 153

-N. edwardsonii, tomato bushy stunt virus, resistance responses, 285

Nodulation

-alfalfa, Rhizobium meliloti, exopolysaccharide EPSI in, ratio of two forms, 755

-alfalfa and soybean, Sinorhizobium spp., Serratia marcescens gene, 748

-in bean, Rhizobium etli metZ gene role, 24

-Bradyrhizobium japonicum on Vigna umbellata, response to mutant and signals, 766

—Lotus japonicus, Agrobacterium rhizogenes in transformation, 275

-Rhizobium etli nolL gene, efficiency and fucosyl residue, 236

-Rhizobium etli on Phaseolus vulgaris, symbiosome differentiation, 515

- Sinorhizobium meliloti on Medicago truncatula, early nodulin gene, 544

- soybeans to Sinorhizobium fredii, Nod factors, 207

Nomura, K., 385

Nordera, P., 391, 401

Norman, C., 640

Nowak, G., 410

Oh, B.-J., 1042

Oh, C., 633

Oláh, B., 755

Olivares, J., 1090

Oliver, R. P., 960

Ollero, F. J., 207

Olliver, R. P., 1051

Oparka, K. J., 894

Ophiostoma novo-ulmi, pathogenicity gene, $O$. ulmi introgression, 6

Ovtsyna, A. L., 252

Paenibacillus polymyxa, in Arabidopsis thaliana, gene change induced by rhizobacterium, 951

Palukaitis, P., 628, 733, 985

Palva, E. T., 575, 640

Panicum mosaic virus

-on pearl millet, panicle infection, cover photo, February

- synergism induced by satellite, 163

Parasponia andersonii, nodulation by Rhizobium, 293

Park, J.-W., 285

Parniske, M., 93

Partridge, J. E., 563

Passeri, F., 45

Patriarca, E. J., 24, 515, 926

Pea (see also Pisum sativum)

- genotype defense genes, fungus and bacterial induction of defense genes, 976

-Nectria haematococca, regulatory signals in culture and pathogenesis, 733

-Rhizobium symbiosomes, synthesized protein origin, 319

Pear, Japanese, Alternaria alternata on, conidial development and melanin biosyntheis gene, 59

Pérez-Espinosa, A., 852

Pernas, M., 624

Perombelon, M. C. M., 499

Peronospora parasitica, on Arabidopsis thaliana, RPP13 locus, mapping, 792

Perotto, S., 785

Peters, N. K., 766, 1082

Phaseolus vulgaris

- acquired resistance, Pseudomonas aeruginosa and salicylic acid role, 450

- anthracnose, ancestral resistance gene cluster, coevolution, 774

-Azorhizobium caulinodans on, Nod factors and chemical substituents, 820

-nodulation and methionine biosynthesis, Rhizobium etli met $Z, 24$

-nodule development and symbiosome differentiation, 515

- Rhizobium etli on roots of, nodule formation, cover photo, June

-Rhizobium etli, trpB gene, symbiosis, 926

Phengrasamy, N. B., 35

Phillips, D. A., 803

Phytoalexin, sorghum, synthesis and chalcone synthase transcripts, 479

Phytophthora infestans, on potato

-cysteine protease gene, 1114

-hypersensitivity genes, 356

Phytotoxins, rhizobitoxine, Rhizobium-legume symbiosis, 1082

Pieterse, C. M. J., 720, 911

Pinel, M., 792

Piotte, C., 585

Pirhonen, M., 575

Pisolithus tinctorius, polypeptides, ectomycorrhiza-regulated cell wall, 862

Pisum sativum (see also Pea)

- arbuscular mycorrhizal fungi, lectinlike glycoprotein, gene encoding, 785

Plum pox virus, on Nicotiana benthamiana, resistance and mitotic stability, 103

Poch, H. L. C., 1016

Pokorny, J., 647

Ponz, F., 1016

Postia placenta, polygalacturonases from, 703
Potato

-blackleg, gene mutation and virulence, 499

-Erwinia carotovora in tubers, maize GapC4 promoter induction, 182

-late blight, cysteine protease gene, resistance, 1114

- Phytophthora infestans, hypersensitivity, genes, 356

- potato cyst nematode and potato virus X resistance, in wild potato species, 197

- snakin-1 isolated from, 16

Potato virus A, on Nicandra physaloides, systemic infection determinants, 1074

Potato virus $\mathrm{X}$

-hypersensitivity, 25-kDa movement protein, 536

-on tobacco, avirulence, Avr 9 and gene inf1, 459

-on wild Solanum species, resistance, 197

Potyvirus plant interaction, molecular biology, review, 367

Primo, J., 227

Prior, D. A. M., 894

Promé, J.-C., 68, 236, 820

Pseudomonas spp.

- $P$. aeruginosa, salicylic acid production, bean resistance, 450

-P. fluorescens, on Arabidopsis thaliana, systemic resistance, 720

-P. syringae: in Arabidopsis, systemic resistance, locus identification, 911; on Arabidopsis thaliana, salicylic acid induction, 1022; on bean, hypersensitivity and calcium-binding protein, 712 ; lipodepsipeptide from, bioactive in planar lipid membranes, 401; lipodepsipeptide toxin interaction, membranes, 391 ; on soybean, heat stress response and dnaK, 563; on tobacco, hypersensitive response and jasmonic acid, 74; on white clover, peroxidase cDNA, characterization, 825

Psophocarpus tetragonolobus, nodulation by Rhizobium, cover photo, April

Pueppke, S. G., 207, 293

Pyrenophora tritici-repentis, on wheat, hostspecific protein toxin, characterization, 728

Rademaker, G.-J., 252

Radutoiu, S. E., 275

Rajamäki, M.-L., 1074

Ralstonia solanacearum, genetic material exchange with host, 467

Ramírez, M., 1008

Ranson, J. C., 969

Rasmussen, S. W., 960

Redman, R. S., 969

Reinhold-Hurek, B., 813

Reis, D., 463

Requena, N., 934

Resistance

-Arabidopsis thaliana to powdery mildew, species comparison, 1051

-Arabidopsis to Pseudomonas, basal and systemic, 911

-Nicotiana edwardsonii to tomato bushy stunt virus, cell-to-cell movement, 285

- potato to late blight, cysteine protease gene, 1114

Revers, F., 367

Reviewer acknowledgment, 3

Rhizobacteria

- Glomus mosseae interaction, 934

-plant-growth-promoting, gene change in Arabidopsis, 951 
Rhizobium spp.: on legume roots, fusicoccin effect on infection, 1090; nested host ranges, 293; on pea, synthesized protein origin,in symbiosomes, 319; on white clover, peroxidase cDNA, characterization, 825

$-R$. etli: on bean, symbiosome differentiation and nodulation, 515; on bean, $\operatorname{trp} B$ gene role in symbiosis, 926; effect on Vitreoscilla sp. hemoglobin, genetic engineering, 1008 ; $m e t Z$ gene, methionine biosynthesis and nodulation, in bean, 24; nolL gene, nodulation efficiency, 236; on Phaseolus vulgaris, nodule formation, cover photo, June

-R. leguminosarum: nodX genes in strains, comparison, 252; on pea, defense genes induced by, 976; siderophore production, gene factor regulation, 994; on Vicia sativa, lipochitin oligosaccharides, 839

- R. meliloti, on alfalfa, exopolysaccharide EPSI in, two forms, 755

Riccio, A., 24, 515, 926

Rice

-Azoarcus sp. on roots of, nif gene expression, 813

-blast, pathogenicity mutants, 129

-wounding, fungal infection, kinase induction, 1064

Rodriguez, R. J., 969

Rodríguez-Palenzuela, P., 624

Rodríguez-Rosales, M. P., 1090

Roldán-Arjona, T., 852

Rolfe, B. J., 252

Romero, D., 236

Rose, M. S., 901

Rosendahl, L., 319

Rosso, M.-N., 585

Roussel, H., 976

Rouster, J., 960

Rubio, T., 87, 153

Ruiz-Lozano, J. M., 976

Ruiz-Rubio, M., 852

Ruiz-Sainz, J. E., 207

Ryals, J., 53

Saenz, G., 1051

Saenz, R., 35

Salánki, K., 1105

Salcedo, G., 624

Salmond, G. P. C., 499

Samac, D. A., 263, 882

Sánchez-Monge, R., 624

Sanjuan, J., 1090

Sanz-Alférez, S., 440

Sauvage, C., 119

Scala, F., 419

Schaffrath, U., 508

Schoelz, J. E., 919

Schoenbeck, M. A., 882

Scholthof, H. B., 87, 153, 285, 670

Scholthof, K.-B. G., 163

Schots, A., 872

Schweizer, P., 647

Segura, A., 16

Serratia marcescens, chitinase gene, effect on Sinorhizobium spp. in soybean and alfalfa nodulation, 748

Seurinck, J., 440

Sévignac, M., 774

Shaul, O., 1000

Shi, L. F., 526

Shintaku, M. H., 628

Shiotani, H., 691

Sicard, D., 774
Siljak-Yakovlev, S., 947

Silva, H., 1031

Simón-Buela, L., 112

Simonet, P., 467

Simonsen, A. C. W., 319

Sinorhizobium spp., on soybean and alfalfa, nodulation affected by Serratia marcescens chitinase gene, 748

$-S$. fredii, GDP-fucose synthesis genes, mutation and soybean nodulation, 207

-S. meliloti: on alfalfa, nitrogen and carbon metabolism, gene map, 526; on alfalfa, Nod factor-inducible molecular marker, cortical cell activation, 604; on alfalfa, nodulation and aspartate aminotransferase 263 ; bioS and LysR-type regulator, 803; on Medicago truncatula, early nodulin gene, 544

Smant, G., 64, 663, 872

Smith-Becker, J. A., 712

Smoker, M., 189

Snoeck, C., 820

Soberón, M., 1008

Solanum tuberosum (see Potato)

Somerville, S., 1051

Sophora davidii, nodulation by Rhizobium, cover photo, April

Sorghum, Cochiobolus heterostrophus, Colletotrichum sublineolum, defense-related genes, 479

Soto, M. J., 1090

Soybean

- bacterial blight, heat stress and dnaK gene, 563

- Cercospora kikuchii, cercosporin transporter, 901

-cyst nematode, endoglucanase secretion, 64

-Heterodera glycines, gene isolation and transcription, 490

-nodulation affected by Serratia marcescens chitinase gene, 748

-nodulation, 53-kDa nodulin, 218

-nodulation, Nod factors and synthesis genes 207

Spaink, H. P., 207, 252, 839

Squash, cucumber mosaic virus, cell-to-cell movement and capsid protein sequence, 628

Stacey, G., 766

Stall, R. E., 35

Stewart, H. E., 1114

Stiekema, W., 197

Stiller, J., 275

Stokkermans, T. J. W., 766, 1082

Straney, D. C., 733

Streit, W. R., 803

Strelkov, S. E., 728

Stuckey, D. B., 994

Sudarshana, M. R., 337

Sussex, I. M., 328

Szécsi, J., 143, 476

Szegletes, Z., 755

Szilassy, D., 1105

Tagu, D., 862

Tak, T., 839

Talbot, N. J., 129

Tanaka, C., 691

Taraporewaia, Z., 247

Taté, R., 24, 515, 926

Tejero-Mateo, P., 207

Temple, S. J., 526

Tephrosia vogelii, nodulation by Rhizobium, cover photo, April

Thio, S. S.-C., 628

Thomas, C. L., 894
Thomas, C. W., 93

Thomas-Oates, J. E., 207, 252

Thorpe, C. J., 499

Tikhonovich, I. A., 252

Timmusk, S., 951

Tobacco (see also Nicotiana spp)

-hypersensitive response, jasmonic acid accumulation, 74

- leaf protoplasts, proliferation by potato cyst nematode, 872

-pathogenesis-related proteins, mycorrhizal role, 1000

Tobacco mosaic virus

—infection sites, 143, 476

-localized acquired resistance and hypersensitivity, salicylic acid, 655

-on pepper, eggplant, and tobacco, coat protein, 247

Tobacco necrosis virus, on bean, hypersensitivity and calcium-binding protein, 712

Tomatinase, Fusarium oxysporum, on tomato new saponinase class, 852

Tomato

- citrus exocortis viroid and tomato mosaic virus on, gentisic acid effect, 227

-Cladosporium fulvum on, $C f-9$ resistance gene, homologues, 93

-Cladosporium fulvum Tnt1 promoter retrotransposon, 592

-root-knot nematode, chorismate mutase, gland specific, 328

-root-knot nematode, LEMMI9 gene and promoter analysis, 440

-Xanthomonas campestris on, avirulence gene avrRxv expression, 35

Tomato bushy stunt virus

-biological activity, nested gene and dosage control, 670

-cell-to-cell movement and resistance responses, 285

-on Nicotiana benthamiana, wild-type virus restoration, 153

Tomato mosaic virus, gentisic acid effect on plant defense, 227

Toms, A. O., 35

Ton, J., 911

Tör, M., 792

Toth, I. K., 499

Toth, R. L., 356

Toxins

-AK, in Japanese pear pathotype of

Alternaria alternata, 691

- syringotoxin, syringomycin, and syringopeptins, from Pseudomonas syringae, 391

Trepp, G. B., 263, 526

Trichoderma harzianum, biocontrol activity, endochitinase-encoding gene, 419

Trieu, A. T., 171

Trinh, T. H., 947

Tsuge, T., 59, 691

Tsujimoto, T., 59

Tsuyumu, S., 385

Turner, J. G., 1051

Turnip mosaic virus, on Arabidopsis thaliana, resistances, 1016

Upchurch, R. G., 901

Valderrama, B., 1008

Valkonen, J. P. T., 1074

van Brussel, A. A. N., 839

van Buuren, M. L., 171 
Vance, C. P., 263, 526, 882

Van der Eycken, W., 440

Vanderleyden, J., 820

van der Voort, C. R., 872

van der Voort, J. R., 197, 872

van der Vossen, E., 197

Van Loon, L. C., 720, 911

van Montagu, M., 440

Van Montagu, M., 68, 440

Vernoud, V., 604

Verreth, C., 820

Verticillium dahliae, on cotton, fungal elicitors in culture cells, 1095

Vhuvaneswari, T. V., 1082

Vian, B., 463

Vicia sativa

-Rhizobium Nod factors, actin filaments, 829

—root nodule primordia, induction, lipochitin oligosaccharides, 839

Vidal, S., 640

Vigna spp.

-V. radiata, Rhizobium symbiosis, rhizobitoxine role, 1082

-V. umbellata, nodulation by Bradyrhizobium japonicum, mutant and signals, 766

Vinardell, J. M., 207

Virus, evolution, host transgene recombination, overview, 87

Vitreoscilla sp., hemoglobin, Rhizobium etli effect on, 1008

Voiblet, C., 862

Volpin, H., 1000

Volrath, S., 53

Wagner, E. G. H., 951

Wang, Gejiao, 490

Wang, Guo-Liang, 1064

Wang, H. L., 337

Wang, X., 64, 663

Wang, Y., 410

Wasternack, C., 74

Weide, R., 459

Weinman, J., 252

Werner, D., 218

Whalen, M. C., 35

Wheat

- powdery mildew, defense-related genes and transient assay system, 647

-PR-1 proteins, genes encoding, 53

- tan spot, host-specific protein toxin, characterization, 728

White clover, peroxidase cDNA,

characterization and expression in bacterial challenges, 825

Whitehead, E., 670
Wilson, I., 1051

Winzer, T., 218

Wong, S.-M., 628

Woo, S. L., 419

Wulff, B. B. H., 93

Xanthomonas spp.

-X. campestris: on pepper, pathogenesisrelated cDNA genes, sequencing, 0751; on soybean, gene mutant as hypersensitivity in nonhosts, 633; on tomato, avirulence gene avrRxv effect, 35

$-X$. citri, on citrus, division enlargement and cell death, 556

Xiao, S., 1051

Yamamoto, M., 691

Yan, Y., 64, 663

Yang, D., 1064

Yang, Z., 430

Yeoman, K. H., 994

Yoo, J.-Y., 633

Yoshioka, H., 263

Yoshioka, K., 1031

Zhang, H., 35

Zhang, L., 680

Zhao, G., 556 